This item is the archived peer-reviewed author-version of:

\title{
LDV measurement of small nonlinearities in flat and curved membranes. A model for eardrum nonlinear acoustic behaviour
}

\section{Reference:}

Gladiné Kilian, Muyshondt Pieter, Dirckx Joris.- LDV measurement of small nonlinearities in flat and curved membranes. A model for eardrum nonlinear acoustic behaviour

AIP conference proceedings / American Institute of Physics - ISSN 0094-243X - Melville, Amer inst physics, 1740(2016), 050005 Full text (Publishers DOI): http://dx.doi.org/doi:10.1063/1.4952669

To cite this reference: http://hdl.handle.net/10067/1350360151162165141 


\title{
LDV Measurement of Small Nonlinearities in Flat and Curved Membranes. A Model for Eardrum Nonlinear Acoustic Behaviour
}

\author{
Gladiné Kilian $^{1, \text { a) }}$, Muyshondt Pieter ${ }^{1, \text { b) }}$, Dirckx Joris ${ }^{1, c)}$ \\ ${ }^{1}$ University of Antwerp, Laboratory of Biophysics and Biomedical Physics, Groenenborgerlaan 171, 2020 Antwerp, \\ Belgium

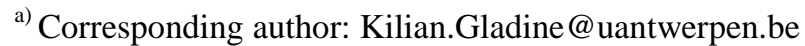 \\ ${ }^{b)}$ Pieter.Muyshondt@uantwerpen.be \\ c) Joris.Dirckx@uantwerpen.be
}

\begin{abstract}
Laser Doppler Vibrometry is an intrinsic highly linear measurement technique which makes it a great tool to measure extremely small nonlinearities in the vibration response of a system. Although the measurement technique is highly linear, other components in the experimental setup may introduce nonlinearities. An important source of artificially introduced nonlinearities is the speaker, which generates the stimulus. In this work, two correction methods to remove the effects of stimulus nonlinearity are investigated. Both correction methods were found to give similar results but have different pros and cons. The aim of this work is to investigate the importance of the conical shape of the eardrum as a source of nonlinearity in hearing. We present measurements on flat and indented membranes. The data shows that the curved membrane exhibit slightly higher levels of nonlinearity compared to the flat membrane.
\end{abstract}

\section{INTRODUCTION}

Literature concerning nonlinear behaviour of the tympanic membrane at acoustic frequencies is rather scarce. In many studies the middle ear response to sound is considered linear up till sound pressure levels of $130 \mathrm{~dB}$. At large quasi-static pressures the human tympanic membrane shows nonlinear behaviour (e.g. Dirckx and Decraemer [1]). From these results one could expect the existence of nonlinear behaviour at acoustic frequencies.

Aerts et al. [2] used multisine sound stimulation and Laser Doppler Vibrometry (LDV) on gerbils and could detect nonlinear response of the tympanic membrane at acoustic frequencies within the sound pressure range of 90 dB SPL to $120 \mathrm{~dB}$ SPL. Recently, similar techniques were used on rabbit and human samples (Peackock et al. [3]). We have used this technique on an anatomically deviating eardrum which had a nearly flat shape. Remarkably, this case showed much less nonlinear behaviour compared to the other normal tent-shaped membranes (Fig. 1). This discrepancy might indicate the importance of the asymmetrical shape of the eardrum as a source of nonlinear behaviour.

Although the LDV setup is highly suitable to measure nonlinearities, the possible nonlinear behaviour of the driving system should not be forgotten. In this study the driving system consists of two earphone speakers. Inside a closed metal cavity they can produce sound pressure levels up to $120 \mathrm{~dB}$. The speakers generate nonlinearities in the stimulation signal which will also be detected in the vibration of the investigated membrane. There are two ways to resolve this problem. The first one is a stimulus correction method [4] and will result in a perfectly sinusoidal stimulation signal for the vibration measurement. The second one is a post-processing correction method which takes into account the nonlinearities in the stimulation signal. Both these methods require a measurement of the stimulation signal and additional information about either the speaker or the investigated membrane. 


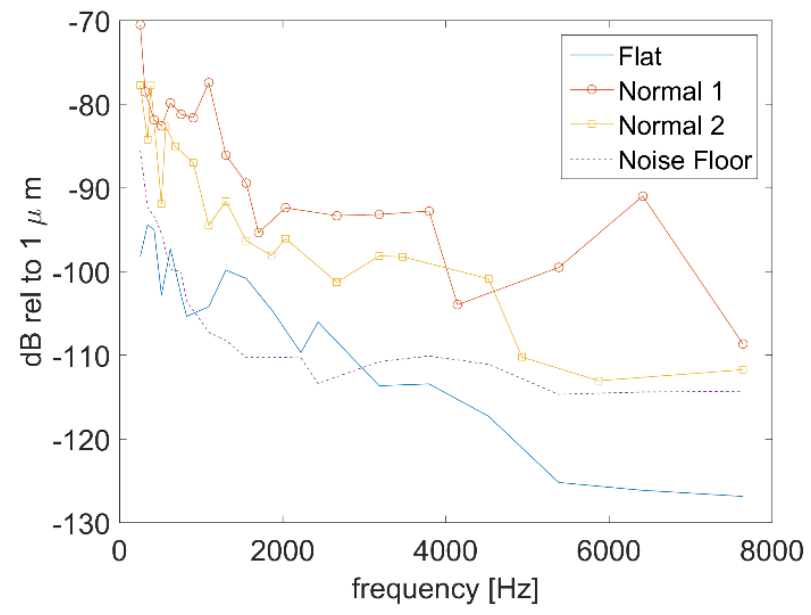

FIGURE 1: Comparison of nonlinear vibration levels for flat and normal eardrums at $108 \mathrm{~dB}$ SPL. The flat eardrum shows significantly less nonlinear behaviour compared to the normal eardrums.

\section{MATERIALS AND METHODS}

\subsection{Experimental Setup}

The experimental setup consists of an LDV (Polytec OFV-5000), Bruël and Kjær probe microphone (type 4182) and earphone speakers. These three elements are connected to a National Instruments card (USB 6251) which is controlled using Matlab scripting. The NI acquisition card its sampling rate is set at $50 \mathrm{kHz}$ and can deliver an output signal with maximal amplitude of 10V.

A closed metal cylindrical cavity was used. The earphone speakers were located at opposite sides of the cylinder and perpendicular to them the probe microphone was inserted. The upper part of the cylindrical cavity was closed by the sample membrane itself. The laser beam of the LDV reached the sample on the outside boundary of the sample membrane. Figure 2 shows a sketch of the experimental setup.

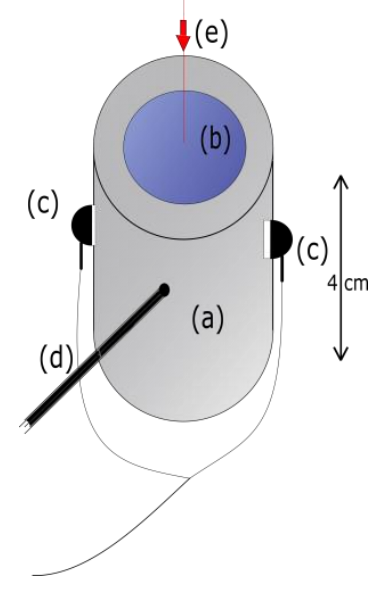

FIGURE 2 Sketch of the experimental setup. (a) Metal cylindrical cavity, (b) membrane sample, (c) Earphone speakers, (d) Probe microphone, (e) Laser beam. 


\subsection{Test Objects}

To investigate the importance of the shape of a membrane with regard to nonlinearity, two different shapes were used in this work. One membrane was flat and another resembled the tent-like shape of a tympanic membrane. Figure 3 shows a cross section along the diameter of the curved membrane. Membranes were clamped at their circumference between a metal ring and the supporting cylinder. The thickness of each membrane was approximately $350 \mu \mathrm{m}$. The freely vibrating part of the membranes had a diameter of $18 \mathrm{~mm}$. A small piece of reflective tape was attached to the surface to increase the reflection of the laser beam directed to the LDV.

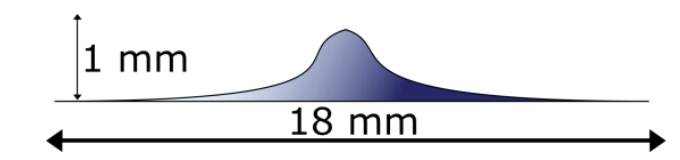

FIGURE 3. Cross section through the non-flat membrane

\subsection{Methods}

\section{Stimulus Correction}

A fine method to avoid nonlinear artefacts at the stimulus is to modify the signal sent to the speaker. Let us suppose that the speaker behaves as nonlinear system which can be described as:

$$
y(x)=x(t)+0.01 x(t)^{2}
$$

$x$ is the voltage sent to the speaker and $y$ is the pressure output recorded by the probe microphone. This pressure output is the stimulus for the vibration measurement done later in the experiment. For simplicity we assume that the frequency response function (FRF) of the speaker is constant as a function of frequency. If $x$ is a sinusoidal function of time, then we get:

$$
y(t)=\sin (2 \pi f t)+\frac{0.01}{2}+\frac{0.01}{2} \sin \left(2 \pi[2 f] t-\frac{\pi}{2}\right)
$$

Thus, $y$ is a signal with frequency components $f$ and $2 f$ which have different phases. The additional signal with frequency $2 f$ is unwanted and should be removed from the pressure output. This is done by modifying the voltage signal $x$ as shown in eq. 3. If the amplitude and phase of $y$ at frequency $2 f$ are known, the signal $x$ can be changed such that the linear component of $y$ produces an additional term that is in antiphase with the $2 f$ component. In this way, the $2 f$ term produced by the quadratic component of $y$ is annihilated.

$$
x^{\prime}=\sin (2 \pi f t)+\frac{0.01}{2} \sin \left(2 \pi[2 f] t+\frac{\pi}{2}\right)
$$

The modified voltage signal $x$ ' eliminates the production of the $2 f$ component but gives rise to undesired higherorder components. These higher-order components have lower amplitudes than the original $2 f$ component and the same procedure can be repeated to eliminate them. This iterative method will result in a stimulation signal $y$ of which the linear component remains at its original amplitude and the nonlinear components are greatly reduced.

The assumption that the speakers FRF is constant as a function of frequency is of course not true. When the voltage signal $(x)$ that is sent to the speaker is converted to pressure waves $(y)$, the phase and amplitude of these waves will be determined by the FRF of the speaker. The amplitude and phase used in $x$ ' should be accommodated using the speakers FRF at the frequency of the harmonic that is being corrected.

\section{Post-Processing Correction}

If no modification is done of the signal that is sent to the speaker, the stimulation signal will contain frequency components which are not intended. The membrane will vibrate at these frequencies and this can be misinterpreted as membrane nonlinear response. If the stimulation signal and the FRF of the membrane at the harmonics of the stimulation frequency are known, a post-processing correction can be done. To remove the nonlinearities caused by 
the stimulus, the Fourier transform of the stimulation signal is multiplied with the FRF of the membrane at the unwanted frequency components and is subtracted from the vibration spectrum. This is a first order correction that works under the assumption that the nonlinearities of the system are small.

\section{Comparison of Membrane Behaviour}

To compare the nonlinear behaviour of a flat and a curved membrane, several measurements were performed. The vibration amplitudes of the harmonics were compared as a function of stimulation amplitude (i.e. SPL), for a fixed stimulation frequency. Alternatively the vibration amplitude of the membrane at the stimulation frequency was used.

Vice versa, the SPL or vibration amplitude at the stimulation frequency can be fixed and we can sweep over a range of frequencies.

\section{General Outline of a Measurement and Signal Creation}

The following procedure was used to generate the stimulation signal and perform a measurement.

1. A sinusoidal voltage signal with frequency $f$ is sent to the earphone speakers

2. A correction factor is calculated to correct either...

a. the effective SPL to the desired SPL

b. the effective vibration amplitude to the desired vibration amplitude at the stimulation frequency

3. A measurement is done of the FRF at the harmonics of $f$ of ...

a. the membrane at a constant SPL for all harmonics (in case of post-processing correction method)

b. the speakers by sending a constant voltage amplitude for all harmonics (in case of stimulus correction method)

i. The speaker is corrected by modifying the voltage signal as described above

4. Fifty measurements are performed, each with a length of $0.1 \mathrm{~s}$

5. The mean value of these fifty measurements is calculated

a. Data-correction is applied if no stimulus correction was done

This is done for every frequency/amplitude level separately.

All signals sent to the earphone speakers were single sine signals. Each signal is extended with 0.05 seconds to eliminate transient effects. When processing the data, the data during this transient time is removed. The frequency $f$ is chosen to be a multiple of 10 . This way an integer number of periods fit into the measured time of 0.1 seconds. As a consequence the Fourier transform can be evaluated exactly at $f$ and its harmonics. 


\section{RESULTS}

\subsection{Correction methods}

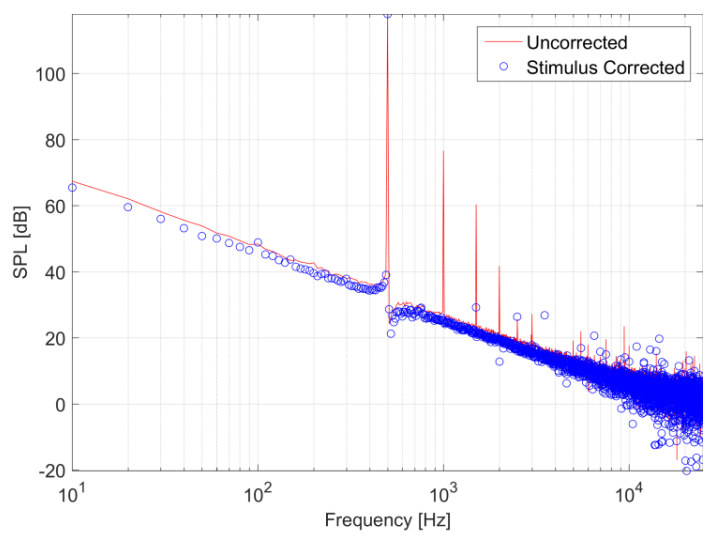

(a)

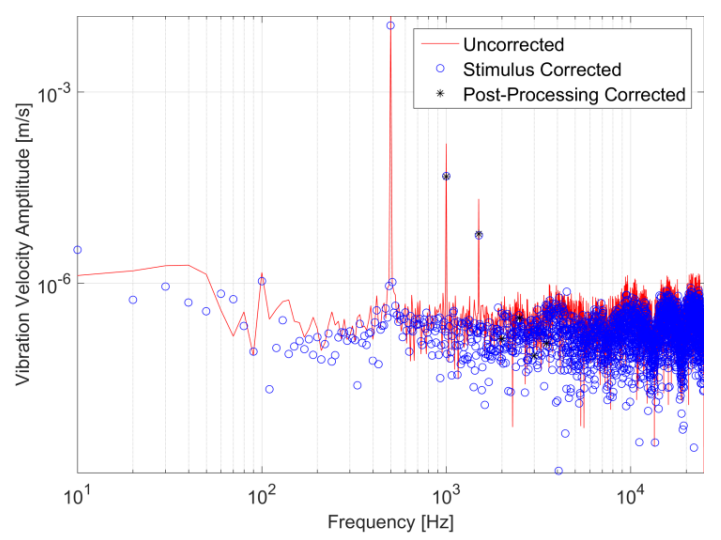

(b)

FIGURE 4 Comparison of the two proposed correction methods, in which corrections were performed for the harmonics of the stimulus frequency of $500 \mathrm{~Hz}$. (a) Measured frequency spectrum of the stimulation signal without and with modification of the signal sent to the speaker. (b) Frequency spectrum of the vibration response of the membrane without any correction (-), with correction of the stimulus (o) and with correction in post-processing $(*)$.

Figure 4 (a) shows the spectrum of the stimulation signal without and with modification of the signal sent to the speaker as described in section '2.3 Methods: Stimulus Correction'. The sound pressure level at the stimulation frequency of $500 \mathrm{~Hz}$ is $110 \mathrm{~dB}$. As expected, the amplitude of the stimulation frequency is the highest peak in the spectrum. The uncorrected stimulus signal also has significant amplitudes at integer multiples of the stimulation frequency. A few other peaks are visible but with small amplitudes. The corrected stimulus signal has the same amplitude at the stimulation frequency but much lower amplitudes at the harmonics. For some frequency components the amplitude has increased after correction. However, these amplitudes are very small as compared to the amplitude at the stimulation frequency and the harmonics of the uncorrected stimulus signal.

Figure 4 (b) shows the spectrum of the vibration velocity of the membrane without and with using stimulus correction and using post-processing correction. For all spectra the vibration velocity amplitude is the highest at the stimulation frequency and the first two higher harmonics. With stimulus correction the peak at the stimulus frequency remains unchanged, but the amplitudes at the higher harmonics are reduced. Similar results are obtained with post-processing correction. The remaining amplitude at the harmonics is now due to nonlinear response of the object, and not caused by nonlinearities in the stimulation signal.

\subsection{Membrane comparison}

The results in this section are obtained using the stimulus correction method. Figure 5 (a) shows the effective vibration amplitude at the stimulation frequency of the flat and curved membrane at $500 \mathrm{~Hz}$ as a function of the desired vibration amplitude at the stimulation frequency. Figure 5 (b) and (c) show the vibration amplitude of the second and third harmonic as a function of the effective vibration amplitude at the stimulation frequency.

A fixed stimulation frequency of $500 \mathrm{~Hz}$ was chosen. Evidently, Fig. 5 (a) shows a straight line with a slope of 1 for both membranes. The vibration amplitude of the second and third harmonic is significantly higher for the curved membrane than for to the flat membrane as seen on Fig. 5 (b) and (c). The second harmonic is dependent on the vibration amplitude at the stimulation frequency and shows a quadratic relation. The relation of vibration amplitude at the stimulation frequency with the third harmonic is not quite clear. 
(a)
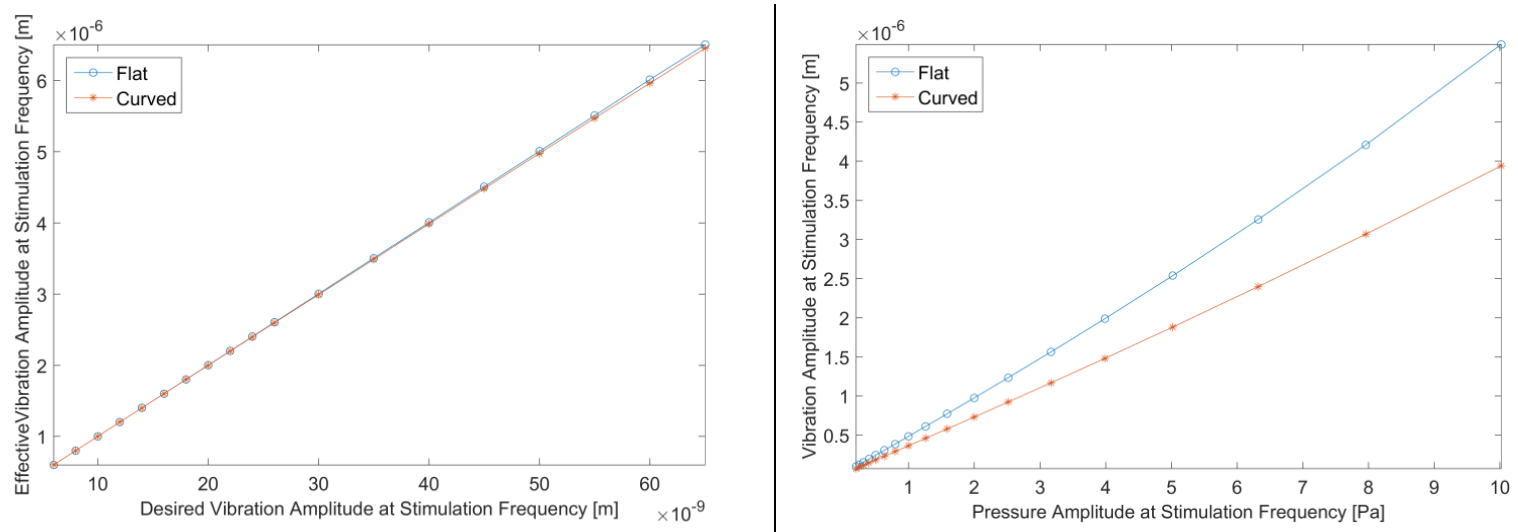

(b)
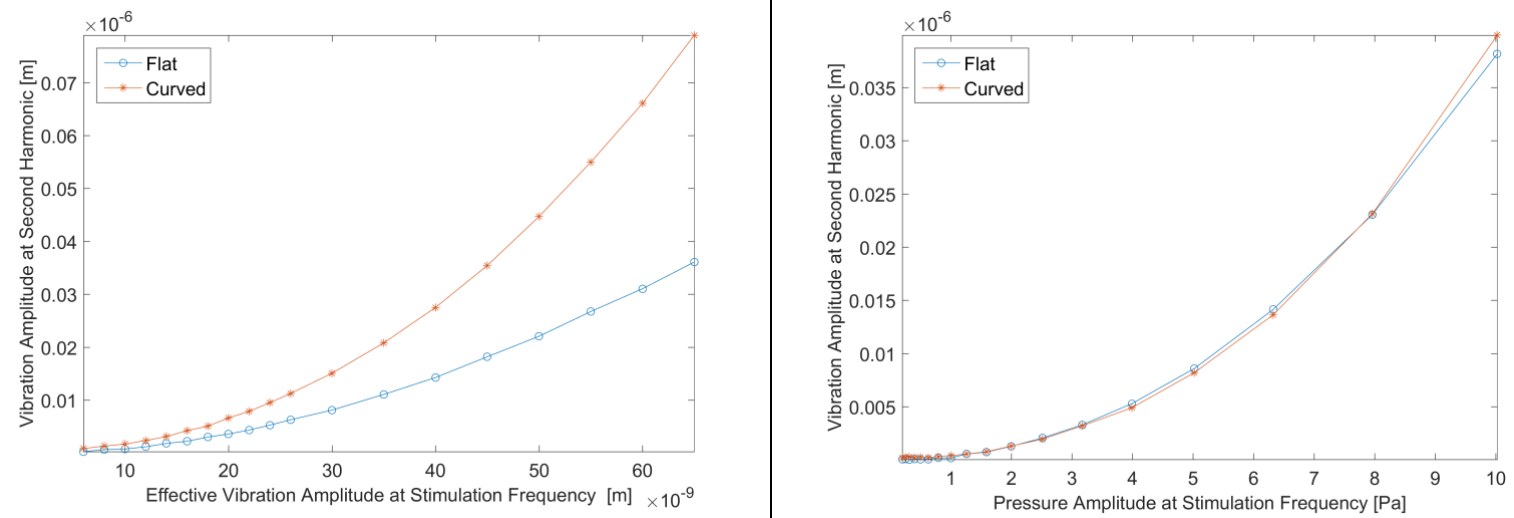

(c)

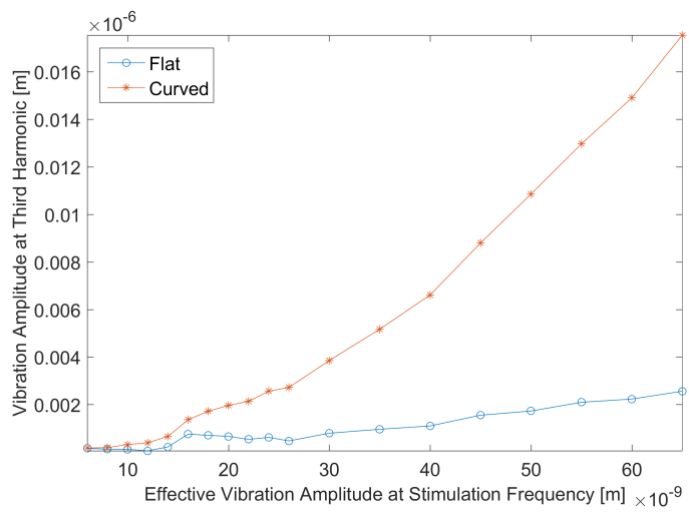

FIGURE 5. Vibration amplitude of the flat membrane (-o-) and the curved membrane (-*-) as a function of vibration amplitude at a stimulus frequency of $500 \mathrm{~Hz}$. Amplitudes are shown for the stimulation frequency (a), second harmonic

(b), and third harmonic (c).

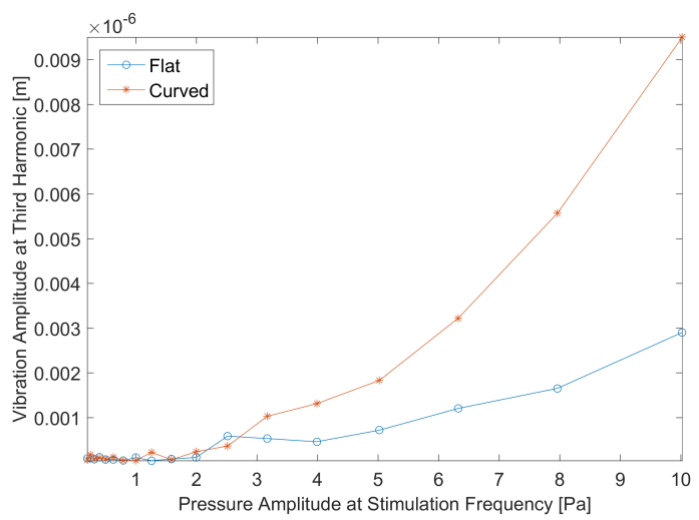

FIGURE 6. Vibration amplitude of the flat membrane ($0-)$ and the curved membrane $\left(-*_{-}\right)$, as a function of pressure amplitude at a stimulus frequency of $500 \mathrm{~Hz}$. Amplitudes are shown for the stimulation frequency (a), second harmonic (b), and third harmonic (c).

Figure 6 shows the membrane vibration amplitude at the stimulation frequency (a), the second harmonic (b) and third harmonic (c) as a function of the pressure, using a stimulation frequency of $500 \mathrm{~Hz}$. In this case the vibration amplitude at the stimulation frequency is not the same for both membranes (Fig. 6 (a)) as it depends on the membrane FRF. At $500 \mathrm{~Hz}$ the vibration amplitude of the flat membrane is larger than that of the curved membrane. Figure 6 (b) shows that the amplitude of the second harmonic is almost equal for both membranes. From Fig. 6 (c) it is clear that the vibration amplitude of the third harmonic is still higher for the curved membrane, despite of the fact that its vibration amplitude at stimulation frequency is smaller than for the flat membrane. 
Figure 7 shows the membrane vibration amplitude of the stimulation frequency (a), second harmonic (b) and third harmonic (c) as a function of stimulation frequency at a fixed sound pressure level. A distinct peak for both membranes is visible (Fig. 7 (a)). The peak frequency of the flat membrane is smaller compared to that of the curved membrane. Multiple peaks are visible in Fig. 7(b) with frequencies equal to the peak frequencies of figure 7(a) but also at half of those frequencies. The curved membrane has an additional peak at a frequency of about $500 \mathrm{~Hz}$. Similarly, Fig. 7 (c) contains peaks at the peak frequencies in Fig. 7 (a) but also at a third of those frequencies although no measurements using sufficiently low stimulation frequencies were performed to clearly visualise this.

Figure 8 shows the membrane vibration amplitude of the stimulation frequency (a), second harmonic (b) and third harmonic (c) as a function of stimulation frequency with fixed vibration amplitude for all stimulation frequencies. Thus the vibration amplitude at the stimulation frequency should be a constant for all stimulation frequencies, which is seen in Fig. 8 (a). In contrast to Fig. 7 (b), Fig. 8 (b) shows no peaks at the peak frequencies of Fig. 7 (a). Peaks are still present at half the peak frequencies of fig. 7(a). For most frequencies higher than these, the amplitude of the curved membrane is larger than that of the flat membrane. This also holds for Fig. 7 (c) but it is less pronounced.

(a)
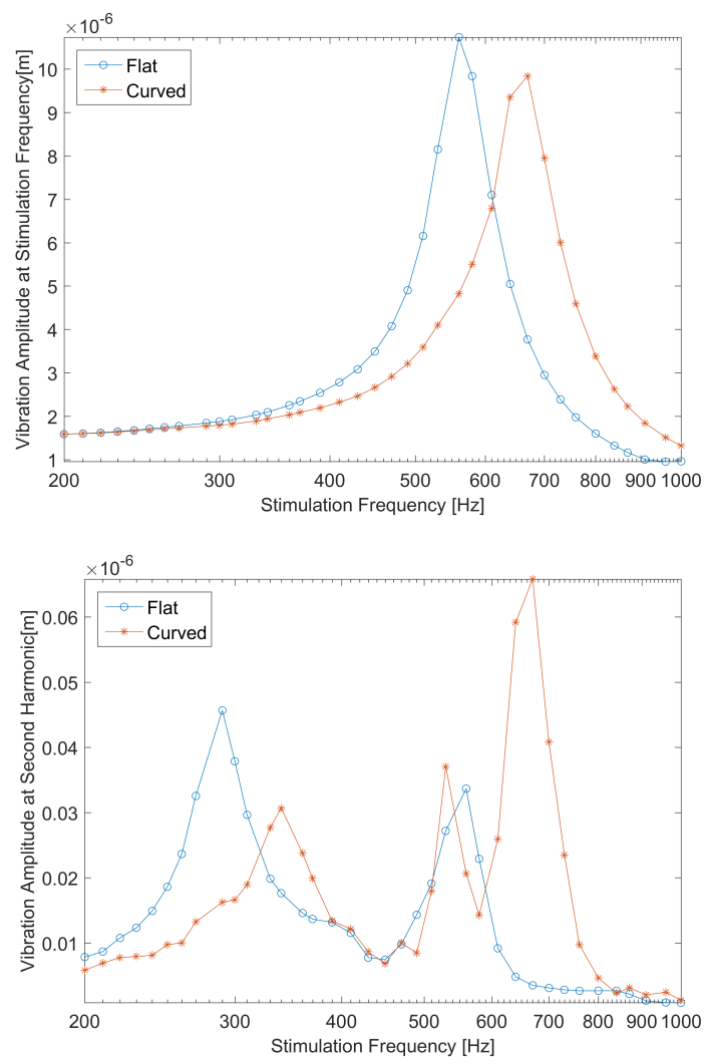
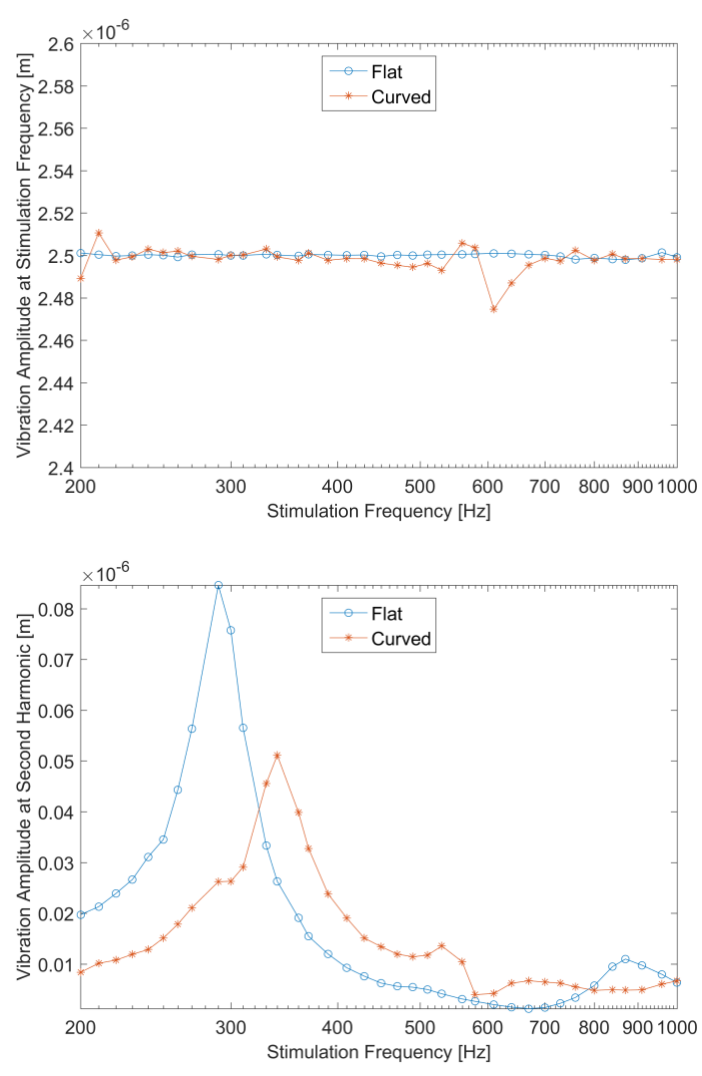


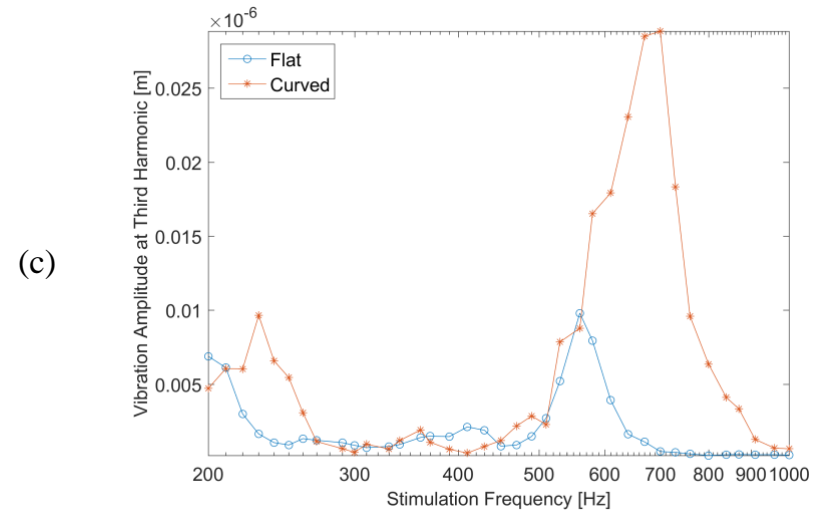

FIGURE 7. Vibration amplitude of the flat membrane (-o-) and the curved membrane $\left(-*_{-}\right)$as a function of stimulus frequency for a fixed SPL. Amplitudes are shown for the stimulation frequency (a), second harmonic (b), and third harmonic (c).

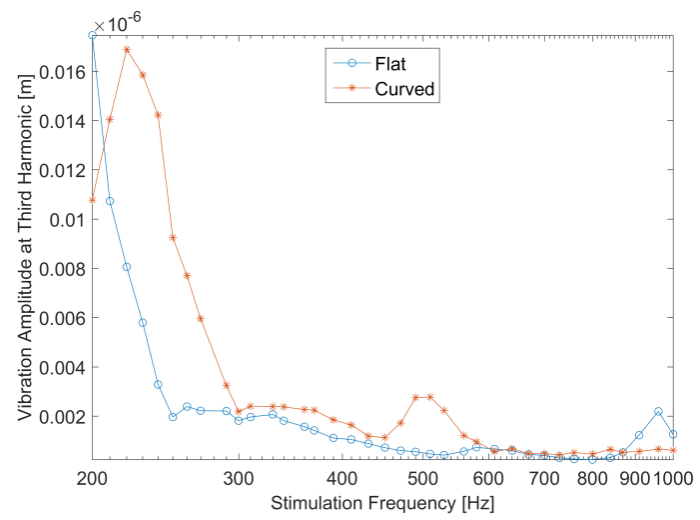

FIGURE 8. Vibration amplitude of the flat membrane (o-) and the curved membrane (-*-) as a function of stimulus frequency for a fixed vibration amplitude. Amplitudes are shown for the stimulation frequency (a), second harmonic (b), and third harmonic (c).

\section{DISCUSSION}

\subsection{Correction Methods}

From Fig. 4 (a) it is clear that sending a modified voltage to the speaker as described in section 'Methods: Stimulus Correction' strongly reduces the amplitude of the harmonics of the stimulation frequency. It works very well for the first higher harmonics but as mentioned before, other higher-order nonlinearities are created. The amplitudes of these nonlinearities are very small and have little influence on the vibration of the membrane.

The harmonics present in the uncorrected stimulus signal contribute to the vibration amplitude of the membrane. This is apparent in Fig. 4 (b), in which the data with corrected stimulus signal and the data corrected using postprocessing show a reduced amplitude at the harmonics. This means that the harmonics of the uncorrected stimulus signal were more or less in phase with the membrane nonlinearities and the corresponding amplitudes add up. Note that the opposite situation might also occur. The results of the stimulus correction method and the post-processing method are in good agreement with each other.

Table 1 lists the pros and cons for both methods.

\begin{tabular}{|c|c|}
\hline Stimulus Correction & Post-Processing Correction \\
\hline $\begin{array}{l}+: \text { Frequency response of the speakers can be measured } \\
\text { quickly since the voltage signal is kept at fixed amplitude }\end{array}$ & $\begin{array}{l}\text {-: Frequency response of the membrane requires } \\
\text { iterative correction to obtain a specific SPL, which takes } \\
\text { longer }\end{array}$ \\
\hline $\begin{array}{l}\text {-: Iterations are needed to modify the voltage signal, this } \\
\text { increases measurement time }\end{array}$ & $+:$ Correction is not done during the measurement \\
\hline $\begin{array}{l}+: \text { If the influence of the nonlinearities becomes significant } \\
\text { this method should still work }\end{array}$ & $\begin{array}{l}\text {-: This is just a first order correction and will not suffice } \\
\text { for high levels of nonlinearity }\end{array}$ \\
\hline
\end{tabular}

TABLE1: Pros and cons of correction methods

It is very important to determine the phase of the stimulation signal at the harmonics as correct as possible. It is the key ingredient to the stimulus correction method because it makes it possible to send an anti-phase signal that annihilates the unwanted frequency components. At higher frequencies the phase changes very rapidly and a small delay can make a big difference in the results of the correction. In this work, not one but two speakers are driven simultaneously. The sound is captured by a single microphone at approximately an equal travelling distance from both speakers. The phases of both speaker signals measured at the tip of the microphone might deviate a bit from one another. For higher frequencies this is more likely to happen and the correction method will work less well than for low frequencies. 


\subsection{Membrane Comparison}

When the voltage signal to the speaker is adapted to obtain a fixed vibration amplitude as a function of frequency, we obtain that the vibration amplitude is the same for both membranes at the stimulation frequency. With this approach, the influence of the frequency response of the membrane on the magnitude of the nonlinearities is removed. This is clearly visible when comparing Fig. 5 (a) and Fig. 6 (a). From Fig. 5 (b) and (c) we can conclude that at $500 \mathrm{~Hz}$ the curved membrane behaves more nonlinearly compared to the flat membrane. The same can be said when looking at Fig. 6 (b) and (c). Although, it is less obvious when examining Fig. 6 (b). Considering that in Fig. 6 (a) the vibration amplitude is larger for the flat membrane, while in Fig. 6 (b) they are almost equal, we can conclude that the quadratic nonlinearity level of the curved membrane is higher. At higher pressures the amplitude of the third harmonic of the curved membrane exceeds the one of the flat membrane.

Figures 5 and 6 display the vibration amplitudes at a single frequency. To draw conclusions about the nonlinear behaviour in general, we need to look at a whole band of frequencies. This information is retrieved from Fig. 7 and 8. Using a fixed pressure value for all stimulation frequencies, the resonance frequency of the membranes is obtained (Fig. 7 (a)). The eigenfrequency of the flat membrane is smaller than that of the curved membrane. This makes sense because the same thickness and materials are used, but the curved membrane has a more complex shape. The influence of resonances at the stimulation frequency is again eliminated by using a fixed vibration amplitude at this frequency (Fig. 8(a)). The effect on the amplitudes of the harmonics is clear when comparing Fig. 7 (b) and (c) with Fig. 8 (b) and (c). The resonances at the eigenfrequencies disappear when the vibration amplitude at the stimulation frequency is fixed. Resonances at half and a third of the eigenfrequency for the second and third harmonic respectively remain visible in both Fig. 7 and 8 . An additional resonance is present in the spectrum of the second and third harmonic of the curved membrane. The origin of this resonance is unknown. From Fig. 8 (b) we can conclude that for a broad range of frequencies the vibration amplitude of the second harmonic is higher for the curved membrane. This is not really the case for the third harmonic (Fig. 8 (c)). It seems that there is a small preference for even nonlinearities as opposed to odd nonlinearities for the curved membrane.

\subsection{Additional Remarks}

Noise estimation Noise floors are not included in the figures. The noise has been estimated by calculating the standard deviation of the complex values of the Fourier transform of the signal. This includes the assumption that the mean of the noise is zero. This may not be entirely true, since some noise sources (such as periodic humming apparatus in the lab) may have specific frequency components.

It would be elegant to filter the data using the phase data of the different measurements. Frequency components of which the phase varies little around a certain value are the result of reproducible signals. These signals must have a physical meaning. Humming machinery signals have a larger standard deviation of the phases, the reason being the transient times imposed between different measurements. These will shift the phase of humming noises but not of stimulation signals.

Ambient noise The stimulation signal captured by the probe microphone might show additional frequency components which come from humming machinery. Probably it is an underestimation of the real noise that these machines produce. The sound measurement is done within the closed cylindrical cavity. Though, the humming noises reach the test object from the outside.

Membrane thickness The membranes have a thickness of approximately $350 \mu \mathrm{m}$. A tympanic membrane is barely $100 \mu \mathrm{m}$ thick. Computer models indicate that through thickness waves produces mainly even nonlinearities and are more important for thick membranes. If thinner membranes were used, even nonlinearities would be reduced and the possible preference for a larger increase of even nonlinearities for curved membranes due to its shape might be more obvious. 


\section{CONCLUSIONS}

In this work two correction methods were investigated to obtain information about the nonlinearity of a system without influence of the nonlinearity of the driving system. These methods consist of correcting the driving system itself during measurement or correcting the data with post-processing. Both methods agree well with each other. Correcting the driving system is more general but does increase the measurement time more. Correcting the data in post-processing is only a first order correction and may be invalid in some cases.

A comparison between a flat and a curved membrane has been made to investigate the importance of membrane shape on nonlinearities. The data shows that the curved membrane exhibits a higher level of nonlinearity compared to the flat membrane. A small preference seems to exist for the second harmonic or the even nonlinearity. The curved membrane is a phantom for the eardrum, which has a similar shape. We will use the model to investigate eardrum shape as a source of nonlinearity in hearing.

\section{REFERENCES}

1. Dirkckx, J.J.J., Decraemer, W.F.S.: Area Change and Volume Displacement of the Human Tympanic Membrane Under Static Pressure. Hearing Research, Vol. 62, No. 1, pp. 99-104, 1992

2. Aerts, J.R.M., Dirckx, J.J.J.: Nonlinearity in Eardrum Vibration as a Function of Frequency and Sound Pressure. Hearing Research, Vol. 263, No. (1-2), pp. 26-32, 2010

3. Peacock, J., Pintelon, R., Dirckx J.J.J.: Nonlinear Vibration Response Measured at Umbo and Stapes in the Rabbit Middle ear. JARO, Vol. 16, No. 5, pp. 569-580, 2015

4. Schoukens, J., Monticelli, L., Rolain, Y.: Identification of Linear Systems in the Presence of Nonlinear Distortions.Periodica Polytechnica Ser. El. Eng. Vol. 36, No. (3-4), pp. 185-196, 1992 